\title{
Intepretações Combinatórias para os Números de Jacobsthal e Jacobsthal-Lucas Generalizados via Ladrilhamentos
}

\author{
Elen V. P. Silva, Cecília P. Andrade, Kenia C. P. Silva \\ UNICAMP- Departamento de Matemática Aplicada \\ Campinas
}

E-mail: elenvps@gmail.com,cpandrade@gmail.com, keycpsilva@gmail.com

\begin{abstract}
Resumo: Neste trabalho vamos apresentar novas interpretações combinatórias utilizando ladrilhamentos, [1, 3, 2], para identidades que envolvem os números de Jacobsthal e Jacobsthal-Lucas, $[5,4]$.
\end{abstract}

Palavras-chave: Números de Jacobsthal Generalizados, Números de Jacobsthal-Lucas Generalizados, Ladrilhamento, Intepretação Combinatória

\section{Definições}

O n-ésimo número de Jacobsthal é definido por

$$
J_{n}=\frac{2^{n}-(-1)^{n}}{3}
$$

generalizando obtemos os números da forma

$$
J_{n}^{s}=\frac{s^{n}-(-1)^{n}}{s+1}
$$

onde $n \geq 0$ é um número natural e $s \geq 0$ é um número real. Ainda podemos definí-los pela relação de recorrência

$$
J_{0}^{s}=0, J_{1}^{s}=1, J_{n}^{s}=(s-1) J_{n-1}^{s}+s J_{n-2}^{s}, n \geq 2 .
$$

O n-ésimo número de Jacobsthal-Lucas é definido por

$$
j_{n}=2^{n}+(-1)^{n},
$$

generalizando obtemos os números da forma 


$$
j_{n}^{s}=s^{n}+(-1)^{n},
$$

onde $n \geq 0$ é um número natural e $s \geq 0$ é um número real. Ainda podemos definí-los pela relação de recorrência

$$
j_{0}^{s}=2, j_{1}^{s}=s-1, j_{n}^{s}=(s-1) j_{n-1}^{s}+s j_{n-2}^{s}, n \geq 2 .
$$

\section{Interpretações}

Considere $a_{n}^{s}$ o $n$-ladrilhamento de ordem $1 \times n$ com quadrados de $s-1$ cores distintas e dominós de $s$ cores distintas. Podemos definir $a_{n}^{s}=J_{n+1}^{s}$, para $n \geq 0$, uma vez que $a_{0}^{s}=J_{1}^{s}=1$, equivalente ao ladrilhamento vazio; $a_{1}^{s}=J_{2}^{s}=s-1$, correspondente aos quadrados de $s-1$ cores distintas, $a_{2}^{s}=J_{3}^{s}=s^{2}-s+1$, correspondente a dominós de $s$ cores distintas adicionado a (s-1)(s-1) correspondente ao 2-ladrilhamento formado por dois quadrados; e assim por diante.

O primeiro resultado abaixo segue da generalização da identidade (1.7) em [4] :

$$
J_{n}^{s}=\sum_{r=0}^{\left\lfloor\frac{n-1}{2}\right\rfloor}\left(\begin{array}{c}
n-1-r \\
r
\end{array}\right) s^{r}(s-1)^{n-1-2 r}
$$

Teorema 1. Para $n \geq 0$, segue que

$$
a_{n}^{s}=\sum_{r \geq 0}\left(\begin{array}{c}
n-r \\
r
\end{array}\right) s^{r}(s-1)^{n-2 r} .
$$

Demonstração: No lado esquerdo da igualdade temos o ladrilhamento de tamanho $n$. Consideremos agora o número de ladrilhamentos de tamanho $n$ com exatamente $r$ dominós. Então, esses ladrilhamentos tem $n-2 r$ quadrados, onde cada um pode ser de $s-1$ cores diferentes. Portanto $n-2 r+r=n-r$ posições a serem preenchidas e assim temos $\left(\begin{array}{c}n-r \\ r\end{array}\right)$ maneiras de escolher em qual posição fica os dominós e $s^{r}$ maneiras de escolha da sua cor. Desta forma, existem $\left(\begin{array}{c}n-r \\ r\end{array}\right) s^{r}(s-1)^{n-2 r}$ ladrilhamentos com exatamente $r$ dominós. (Observermos que $r$ não ultrapassa o valor de $\lfloor n / 2\rfloor$.) Somando em $r$ obtemos o resultado desejado.

Através da identidade

$$
\sum_{k=2}^{n} J_{k}^{s}(s-1)^{n-k}=\frac{J_{(n+2)}^{s}-(s-1)^{n-1}\left(s^{2}-s+1\right)}{s}
$$

fazendo $k=k-1$ e $n=n+1$ segue que 


$$
\sum_{k=1}^{n} J_{k+1}^{s}(s-1)^{n-k}=\frac{J_{(n+3)}^{s}-(s-1)^{n}\left(s^{2}-s+1\right)}{s}
$$

ou ainda,

$$
\sum_{k=1}^{n} J_{k+1}^{s}(s-1)^{n-k}=\frac{J_{(n+3)}^{s}-(s-1)^{n+2}-s(s-1)^{n}}{s}
$$

Mas temos para $k=0, s \sum_{k=0}^{0} J_{k+1}^{s}(s-1)^{n-k}=s(s-1)^{n}$ e, portanto, temos o seguinte resultado.

Teorema 2. Para todo $n \geq 0$,

$$
s \sum_{k=0}^{n} a_{k}^{s}(s-1)^{n-k}=a_{n+2}^{s}-(s-1)^{n+2}
$$

Demonstração: O lado direito da igualdade (5) é o número de (n+2)-ladrilhamentos com quadrados de $s-1$ cores distintas e dominós de $s$ cores distintas, excluindo o ladrilhamento contendo apenas quadrados .

Considere agora a localização do último dominó. Existem $s(s-1)^{n-k} a_{k}^{s}$ ladrilhamentos onde o último dominó cobre as células $k+1$ e $k+2$, uma vez que as células de 1 a $\mathrm{k}$ são ladrilhadas de $a_{k}^{s}$ maneiras, as células $k+1$ e $k+2$ são cobertas por um dominó de uma das $s$ cores disponíveis, e as células de $k+3$ até $n+2$ são cobertas por quadrados de $(s-1)^{n-k}$ maneiras. Portanto, o número de ladrilhamentos com pelo menos um dominó é $s(s-1)^{n-k} a_{0}^{s}+s(s-1)^{n-k} a_{1}^{s}+\ldots+s(s-1)^{n-k} a_{n}$, ou equivalentemente $s \sum_{k=0}^{n} a_{k}^{s}(s-1)^{n-k}$.

Teorema 3. Para todo $n \geq 0$,

$$
a_{m}^{s} a_{n+1}^{s}+s a_{n}^{s} a_{m-1}^{s}=a_{m+n+1}^{s}
$$

Demonstração: O lado direito da igualdade (6) é o número de $(\mathrm{m}+\mathrm{n}+1)$-ladrilhamentos. Consideremos agora as condições de decomposição de um ladrilhamento. Se um $(m+n+1)-$ ladrilhamento é separável na célula $m$, então podemos criar um m-ladrilhamento seguido de um $(n+1)$-ladrilhamento. Existem $a_{m}^{s} a_{n+1}^{s}$ números de termos um $(m+n+1)$-ladrilhamento separável na célula $m$.

Caso contrário, existirá um dominó cobrindo a células $m$ e $m+1$. Então podemos criar um $(m-1)$-ladrilhamento seguido de um dominó e um $(n)$-ladrilhamento. Isso pode ser feito de $s a_{n}^{s} a_{m-1}^{s}$ maneiras. Desde que, não é possível um ladrilhamento ser separável e não separável na célula $m$, simultaneamente, existem $a_{m}^{s} a_{n+1}^{s}+s a_{n}^{s} a_{m-1}^{s}$ ladrilhamentos de tamanho $(m+n+1)$. 
Corolário 4. Para todo $n \geq 0, a_{n}^{s}$ divide $\sum_{i=0}^{n} s^{n-i} a_{2 i}^{s}$.

Teorema 5. Para todo $n \geq 1$,

$$
\left(a_{n}^{s}\right)^{2}=a_{n+1}^{s} a_{n-1}^{s}+(-1)^{n} s^{n}
$$

Demonstração: Temos $\left(a_{n}^{s}\right)^{2}$ ladrilhamentos para um par de n-ladrilhamentos. Também temos $a_{n+1}^{s} a_{n-1}^{s}$ ladrilhamentos para um $(n+1)$-ladrilhamento e um $(n-1)$-ladrilhamento (colocados um sobre o outro). Vamos dividir a prova em dois casos dependendo da paridade de n. Se $n$ é ímpar, então existe pelo menos um quadrado nos dois ladrilhos e portanto o par de nladrilhamentos contém uma falha e consequentemente um cauda. Portanto, trocando as caudas do ladrilhamento obtemos um $(n+1)$-ladrilhamento e um $(n-1)$-ladrilhamento com as mesmas falhas. Essa correspondência é biunívoca entre todos os pares de n-ladrilhamentos contendo falhas e todos os pares de $(n+1)$-ladrilhamento e $(n-1)$-ladrilhamento . No entanto devemos descontar os pares de $(n+1)$-ladrilhamento e $(n-1)$-ladrilhamento contendo apenas dominós, pois estes não contém falhas. Ou ainda, devemos descontar os $s^{\left\{\frac{n+1}{2}+\frac{n-1}{2}\right\}}=s^{n}$ ladrilhamentos formados apenas por dominós de $s$ cores distintas. Logo temos $\left(a_{n}^{s}\right)^{2}=a_{n+1}^{s} a_{n-1}^{s}-s^{n}$.

Quando $n$ é par, aplicamos o mesmo argumento anterior para obter uma correspondência biunívoca entre todos os pares de $n$ - ladrilhamentos e todos os pares de $(n+1)$-ladrilhamento e $(n-1)$-ladrilhamento contendo falhas. Nesse caso, o ladrilhamento contendo apenas dominós é o único ladrilhamento de um par de $n$-ladrilhamentos sem falhas. Logo temos que descontar os $s^{n}$ ladrilhos de $\left(a_{n}^{s}\right)^{2}$. Assim temos $\left(a_{n}^{s}\right)^{2}-s^{n}=a_{n+1}^{s} a_{n-1}^{s}$, o que completa a prova.

Agora vamos dar uma interpretação combinatória para os números $j_{n}^{s}$ de Jacobsthal-Lucas Generalizado. Para tanto defina $j_{n}$ como a quantidade de braceletes com $n$ posições rotuladas no sentido horário, usando quadrados de $(s-1)$ cores e dominós "curvos" de $s$ cores distintas.

Lema 6. Para todo $n \geq 1$,

$$
j_{n}^{s}=a_{n}^{s}+s a_{n-2}^{s} .
$$

Demonstração: Existem $j_{n}^{s} n$-braceletes e estes são de dois tipos: em fase ou fora de fase. Dado um $n$-bracelete em fase, podemos “esticá-lo" em um $n$-ladrilhamento, logo existem $a_{n}^{s} n$ braceletes em fase. Para o caso de um bracelete fora de fase, podemos remover o dominó que cobre as cèlulas $n$ e 1 e portanto obtemos um ladrilhamento de comprimento $n-2$. Portanto, como existem $s$ possibilidades de cores para este dominó, o número de $n$-braceletes fora de fase é $s a_{n-2}^{s}$, e portanto segue o resultado. 
Teorema 7. Para todo $n \geq 1$

$$
j_{n}^{s}=\sum_{r \geq 0} \frac{n}{n-r}\left(\begin{array}{c}
n-r \\
r
\end{array}\right) s^{r}(s-1)^{n-2 r} .
$$

Demonstração: Do lado esquerdo da igualdade (9) temos braceletes de tamanho $n$. Por outro lado, considere braceletes com exatamente $r$ dominós. Com a mesma argumentação do lema (6) temos que existem $\left(\begin{array}{c}n-r \\ r\end{array}\right) s^{r}(s-1)^{n-2 r}$ braceletes em fase e $\left(\begin{array}{c}n-r-1 \\ r-1\end{array}\right) s^{r}(s-1)^{n-2 r}$ braceletes fora de fase. Uma vez que

$$
\left(\begin{array}{c}
n-r-1 \\
r-1
\end{array}\right) s^{r}(s-1)^{n-2 r}=\frac{r}{n-r}\left(\begin{array}{c}
n-r \\
r
\end{array}\right) s^{r}(s-1)^{n-2 r}
$$

temos, somando em $r$, que o número de braceletes de tamanho $n$ é dado por

$$
\begin{gathered}
\sum_{r \geq 0}\left[\left(\begin{array}{c}
n-r \\
r
\end{array}\right) s^{r}(s-1)^{n-2 r}+\frac{r}{n-r}\left(\begin{array}{c}
n-r \\
r
\end{array}\right) s^{r}(s-1)^{n-2 r}\right]= \\
\sum_{r \geq 0} \frac{n}{n-r}\left(\begin{array}{c}
n-r \\
r
\end{array}\right) s^{r}(s-1)^{n-2 r}
\end{gathered}
$$

como desejado.

Teorema 8. Para todo $n \geq 0$,

$$
(s+1)^{2} a_{n}^{s}=j_{n+2}^{s}+s j_{n}^{s}
$$

Demonstração: Considere $S_{1}$ o conjunto contendo $(s+1)^{2}$ cópias de cada $n$-ladrilhamento e $S_{2}$ o conjunto formado pelos $(n+2)$ - braceletes ou duas cópias de cada $n$ - bracelete. Temos que $\left|S_{1}\right|=(s+1)^{2} a_{n}$ e $\left|S_{2}\right|=j_{n+2}+2 j_{n}$. Para provar essa identidade vamos estabeler 1-9 correspondência entre os conjuntos $S_{1}$ e $S_{2}$.

Dado um $n$-ladrilhamento podemos criar naturalmente 4 tipos de braceletes:

1.A quantidade de $(s-1)(s-1)(n+2)$ - braceletes em fase terminados em dois quadrados inseridos;

2.A quantidade de $s(n+2)$ - braceletes em fase terminados em um dominó inserido;

3.A quantidade de $s(n+2)$ - braceletes fora de fase com um dominó inserido nas células $(n+2,1)$

4. (n)-bracelete em fase concatenando as células $n$ e 1 . 
Ainda estão faltando dois tipos de braceletes: $(n)$-braceletes fora de fase e $(n+2)$-braceletes em fase terminados em quadrado com um dominó inserido cobrindo o par de célula $(n, n+1)$. Note que a construção de tais braceletes depende do $n$-ladrilhamento tomado.

Se o $n$-ladrilhamento termina em quadrado então criamos um $(n+2)$ - bracelete em fase terminado em quadrado inserindo um dominó cobrindo o par de célula $(n, n+1)$. Teremos então $s(n+2)-$ braceletes desta forma.

Se o $n$-ladrilhamento termina em dominó então criamos um $(n)$-bracelete fora de fase rotacionando o bracelete em uma célula no sentido horário. Tomando $s$ cópias deste bracelete teremos $s$ cópias do $n$-ladrilhamento terminado em dominó, que adicionado as $s$ cópias do $n$-ladrilhamento terminado em quadrado necessárias para fazer o $(n+2)$ - bracelete em fase terminado em quadrado resultam em $s$ cópias de $a_{n}$.

No entanto, para termos o total de $n$ - braceletes, $j_{n}$, é necessário tomar $s$ cópias do bracelete do tipo descrito no item 4. Assim, teríamos o total de $j_{n+2}^{s}+s j_{n}^{s}$ braceletes sendo levados bijetivamente a $(s-1)(s-1)+s+s+s+s=(s+1)^{2}$ cópias de cada $n$-ladrilhamento.

Utilizandos os mesmos argumentos demonstramos, via ladrilhamento, as seguintes identidades generalizadas encontradas a partir das identidades dadas em [4].

Teorema 9. Para todo $n \geq 0$,

$$
s \sum_{i=0}^{n} j_{i}^{s}(s-1)^{n-k}=j_{n+2}^{s}-(s-1)^{n+2}
$$

Teorema 10. Para todo $n \geq 0$,

$$
j_{n+1}^{s} a_{n}^{s}=a_{2 n+1}^{s}
$$

Teorema 11. Para todo $n \geq 0$,

$$
(s-1) a_{n-1}^{s}+j_{n}^{s}=2 a_{n}^{s}
$$

Teorema 12. Para todo $n \geq 0$,

$$
j_{2 n+1}^{s}=(s-1) \sum_{i \geq 0} s^{n-i} j_{2 i}^{s}
$$

Teorema 13. Para todo $n, m \geq 0$,

$$
j_{m}^{s} j_{n}^{s}+(s+1)^{2} a_{m-1}^{s} a_{n-1}^{s}=2 j_{m+n}^{s}
$$

Teorema 14. Para todo $n \geq 0$ temos

$$
a_{n-1}+a_{n}=s^{n}
$$


Teorema 15. Para todo $n \geq 0$,

$$
a_{2 n+1}^{s}=\sum_{i=0}^{n}(s-1) s^{n-i} a_{2 i}^{s} .
$$

Notemos que todas as identidades em [4] podem ser vistas como corolários das identidades generalizadas e portanto podem também ser demonstradas via ladrilhamento.

\section{Referências}

[1] Benjamin, A. T., Plott, S. S., Sellers, J.A. Tiling Proofs of Recent Sum Identities Involving Pell Numbers Annals of Combinatoric, 12(3), 271-278 (2008)

[2] Benjamim, A. T., Quinn, J. J. Proofs that Really Count: The Art of Combinatorial Proof. The Dolciani Mathematical Expositions, 27, Mathematical Association of America, Washington, DC, 2003.

[3] Briggs, K. S., Little, D. P., Sellers, J.A. Combinatorial Proofs of Various q-Pell Identities via Tilings Annals of Combinatoric, 14, 407-418 (2010)

[4] Horadam, A. F. Jacobsthal Representation Numbers. The Fibonacci Quarterly 34.1 (1996): 40-54.

[5] Sloane, N. J. A. The On-line Encyclopedia of Integer Sequences, published eletronically at https://oeis.org/. 